\title{
SIMPLIFIED IMPACT ANALYSIS IN FENICS USING HERTZ CONTACT LAW
}

\author{
JAROSLAV SCHMIDT \& TOMÁŠ JANDA \\ Department of Mechanics, Czech Technical University in Prague, Czech Republic
}

\begin{abstract}
The interaction of two bodies during and after impact results in complex behavior. Modelling such behaviour can be simplified by introducing a closed-form law, e.g. the Hertz law for frictionless contact. Such a model significantly reduces implementation challenges because the kinematic constraint of impenetrability is replaced by nonlinear force. This paper investigates such impact models focusing mainly on implementation details in the nowadays popular finite element solver FEniCS. This solver provides a high level of abstraction for PDEs description and solution techniques. The process of matrix assembling and localization is automated by the solver. The contribution shows usability of the FEniCS for Hertz law implementation and investigates the influence of the boundary conditions. Although the contribution focuses mostly on implementation details, example models correspond to real problem of glass plates subjected to low-velocity impact and are validated by experimentally obtained results.

Keywords: Hertz law, impact, FEniCS.
\end{abstract}

\section{INTRODUCTION}

The contribution partially follows objectives of Czech Science Foundation grant No. 19$15326 \mathrm{~S}$, which is focused on design and advanced modeling of forced-entry and bullet resistant glass structures under low velocity impact. Glass is fragile material suffering from progressive collapse. Laminated glass was invented to overcome this difficulties. It consist of several glass plates binded together by thin viscous interlayers. The invention of this composite has spread out in the automotive industry as material for windshields. Later civil engineering starts using laminated glass for structural elements, e.g. stairs, railings, awnings or facade parts. All these elements are required to withstand loading configurations including, e.g., seismic events, extreme winds and other climatic or fire exposures, impacts of hard or soft elements, or blast loads. Hence the pre- and post-breakage behavior of laminated glass must be investigated to safely design elements dimensions. More information about laminated glass behavior and glass structure design can be found in [1].

Impact is complicated mechanical process generally involving the chaos theory. Fortunately, from the engineering point of view, the impact between two bodies can be described by simplified constitutive relationship. It leads to the idea of introducing a nonlinear force between bodies. Example of such constitutive equation is the Hertz law [2], which is used in this study.

Nowadays phase-field damage models [3] are popular for brittle fracture modeling due to their easy-to-implement formulation without extra ad-hoc criteria. Governing equations can be solved using common finite element method (FEM), such as library FEniCS [4] with python interface. In this paper, we therefore investigate implementation of Hertz law in FEniCS library for solid glass without damage which serves as preparation for future phasefield implementation for laminated glass.

\section{FORMULATION}

The FEniCS library is open-source platform for solving set of partial differential equations (PDEs), which provides abstract python interface. The main input for calculation is the weak form of governing equations supplemented by relevant boundary conditions. For this 
reason, we recall the basic linear elasticity in both, weak (integral) and strong (differential) form. The following equations are well-known continuum mechanics relationships, recall for example [5].

Response of elastic body $\Omega$ with boundary $\partial \Omega=\Gamma=\Gamma_{\mathrm{f}} \cup \Gamma_{\mathrm{u}}$ is described by vector displacement field $\mathbf{u}(t)$ which at each time instant fulfils the equilibrium condition

$$
\operatorname{div} \boldsymbol{\sigma}+\mathbf{b}=\rho \ddot{\mathbf{u}} \quad \text { in } \Omega
$$

supplemented by boundary conditions

$$
\begin{aligned}
\mathbf{u}=\overline{\mathbf{u}} & \text { on } \Gamma_{\mathrm{u}}, \\
\boldsymbol{\sigma} \cdot \mathbf{n}=\overline{\mathbf{f}} & \text { on } \Gamma_{\mathrm{f}},
\end{aligned}
$$

where $\boldsymbol{\sigma}(\mathbf{u})$ is Cauchy stress tensor, $\mathbf{b}$ is body force, $\rho$ is density, $\overline{\mathbf{u}}$ is prescribed Dirichlet displacement field on boundary $\Gamma_{\mathrm{u}}, \overline{\mathbf{f}}$ is prescribed Neumann force on boundary $\Gamma_{\mathrm{f}}$ and $\mathbf{n}$ is outward unit normal. Constitutive relationship is given by Hooke's law using Lame's constant $\lambda$ and $\mu$ as

$$
\boldsymbol{\sigma}(\mathbf{u})=\lambda \operatorname{div}(\mathrm{u}) \mathbf{I}+2 \mu \varepsilon,
$$

where $\mathbf{I}$ is unit tensor and finally $\varepsilon$ is linearized strain

$$
\varepsilon=\frac{1}{2}\left(\nabla \mathbf{u}+(\nabla \mathbf{u})^{\top}\right)
$$

However, strong form requires excessive regularity of solution. Somehow more natural is to formulate the problem integrally in the weak form. This form is obtained by multiplying strong form by test function. After integration over domain and applying divergence theorem we get the weak form. It states: find $\mathbf{u}(t)$ which satisfy Dirichlet boundary conditions such that

$$
\int_{\Omega} \boldsymbol{\sigma}: \delta \varepsilon \mathrm{d} V+\int_{\Omega} \rho \ddot{\mathbf{u}} \cdot \delta \mathbf{u} \mathrm{d} V=\int_{\Omega} \mathbf{b} \cdot \delta \mathbf{u} \mathrm{d} V+\int_{\Gamma_{\mathrm{f}}} \mathbf{f} \cdot \delta \mathbf{u} \mathrm{d} S
$$

for all $\delta \mathbf{u}$ vanishing on boundary $\Gamma_{\mathrm{u}}$.

This equation governs the response of elastic impacted body. Besides we must also describe position of the rigid-body impactor denoted as $u_{0}(t)$. Its motion is modeled by one degree of freedom [6] with mass $m_{0}$, therefore it must satisfy scalar equation of motion

$$
m_{0} \ddot{u}_{0}=F_{\mathrm{c}}\left(u_{0}-u_{\mathrm{hit}}\right)
$$

where $u_{\text {hit }}$ is scalar value of plate displacement at the point of impact in direction of impactor movement $u_{0}$. Force $F_{\mathrm{c}}$ is nonlinear force given by Hertz law

$$
F_{\mathrm{c}}(\alpha)=k_{0}\langle\alpha\rangle^{3 / 2}
$$

where $k_{0}$ is contact stiffness and $\langle\alpha\rangle$ is positive part of $\alpha$, symbolically

$$
\langle\alpha\rangle=\frac{|\alpha|+\alpha}{2} .
$$

The same force $F_{\mathrm{c}}\left(u_{0}-u_{\text {hit }}\right)$ with opposite direction acts on elastic body as nonlinear contribution in surface force $f$. This forces bind together response of elastic medium with 
one-DoF impactor. Without loss of generality we assume that no other surface forces are prescribed and $\mathbf{b}=\mathbf{0}$.

Spatial discretization of (6) is provided by finite element method through FEniCS [4]. Distribution of displacement function $\mathbf{u}$ is approximated by selected base functions defined on small compact subsets of $\Omega$. After spatial integration, discretized version of equilibrium equations is obtained as matrix equation

$$
\left[\begin{array}{cc}
\boldsymbol{M} & \mathbf{0} \\
\mathbf{0} & m_{0}
\end{array}\right]\left[\begin{array}{c}
\ddot{\boldsymbol{u}}(t) \\
\ddot{u}_{0}(t)
\end{array}\right]+\left[\begin{array}{cc}
\boldsymbol{K} & \mathbf{0} \\
\mathbf{0} & 0
\end{array}\right]\left[\begin{array}{c}
\boldsymbol{u}(t) \\
u_{0}(t)
\end{array}\right]=\left[\begin{array}{c}
-\boldsymbol{a} F_{\mathrm{c}}\left(u_{0}-u_{\mathrm{hit}}\right) \\
F_{\mathrm{c}}\left(u_{0}-u_{\mathrm{hit}}\right)
\end{array}\right],
$$

where $\boldsymbol{K}$ and $\boldsymbol{M}$ are stiffness and mass matrices of elastic body and $\boldsymbol{a}$ is localization vector which distributes nonlinear Hertz force to corresponding nodes. Matrix eqn (10) represents the set of nonlinear equations. Nonlinearity arises due to force $F_{\mathrm{c}}$ and positive-part operator. Above that this force is not continuously differentiable. It can bring numerical difficulties when smooth Newton method is used. Nevertheless this discrepancy is negligible in most of engineering problems with sufficiently small time step. In our cases, no such numerical difficulties appeared.

The FEniCS library may be advantageously used for solving eqn (10). Individual integrals from (6) are stated using the FEniCS' Unified Form Language (UFL) in python environment and localization and assembling process is performed by library itself. This brings a higher level of abstraction above the problem under consideration.

Extension of model can be simply implemented by modifying the weak form. For example, flexible supporting of edges at the part of boundary $\Gamma_{\mathrm{k}}$ is implemented by adding following member to weak form

$$
\int_{\Gamma_{\mathrm{k}}} k(\mathbf{u}-\overline{\mathbf{u}}) \cdot \delta \mathbf{u} \mathrm{d} S
$$

This is the same as enforcing the Dirichlet boundary conditions by penalties, with the difference that now we do not select the parameter $k$ as large as possible, but $k$ has the physical meaning of the stiffness of the support. In some experiments, the impacted plate is put on a flexible pad without additional anchoring. Plate is supported only in one direction in this case, whereas movement in other direction is allowed. It corresponds with the weak contribution

$$
\int_{\Gamma_{\mathrm{k}}} k\langle\mathbf{u}-\overline{\mathbf{u}}\rangle \cdot \delta \mathbf{u} \mathrm{d} S .
$$

Recall that $\langle x\rangle$ is positive part operator, which is applied element-wise on vector argument.

Similarly the damping of boundary conditions can be added to model. Viscous damping is dissipative phenomena without variational structure in the meaning, that there is no potential. Nevertheless the damping can be added directly to weak form as following ad-hoc integral expression

$$
\int_{\Gamma_{\mathrm{c}}} c \dot{\mathbf{u}} \cdot \delta \mathbf{u} \mathrm{d} S
$$

where $c$ is boundary damping coefficient. It this case the governing matrix equation has following form

$$
\left[\begin{array}{cc}
\boldsymbol{M} & \mathbf{0} \\
\mathbf{0} & m_{0}
\end{array}\right]\left[\begin{array}{c}
\ddot{\boldsymbol{u}}(t) \\
\ddot{u}_{0}(t)
\end{array}\right]+\left[\begin{array}{cc}
\boldsymbol{C} & \mathbf{0} \\
\mathbf{0} & 0
\end{array}\right]\left[\begin{array}{c}
\dot{\boldsymbol{u}}(t) \\
\dot{u}_{0}(t)
\end{array}\right]+\left[\begin{array}{cc}
\boldsymbol{K} & \mathbf{0} \\
\mathbf{0} & 0
\end{array}\right]\left[\begin{array}{c}
\boldsymbol{u}(t) \\
u_{0}(t)
\end{array}\right]=\left[\begin{array}{c}
-\boldsymbol{a} F_{\mathrm{c}}\left(u_{0}-u_{\mathrm{hit}}\right) \\
F_{\mathrm{c}}\left(u_{0}-u_{\mathrm{hit}}\right)
\end{array}\right]
$$


Again, the matrix $C$ and its components are assembled by FEniCS library.

\section{IMPLEMENTATION}

In this section we recall critical parts of implementation. The dimension of matrices (number of DoFs) is driven by FEniCS through abstract function spaces. As demonstrated by eqn (14) we need to extend the number of DoFs by one, It can be done in FEniCS as follows

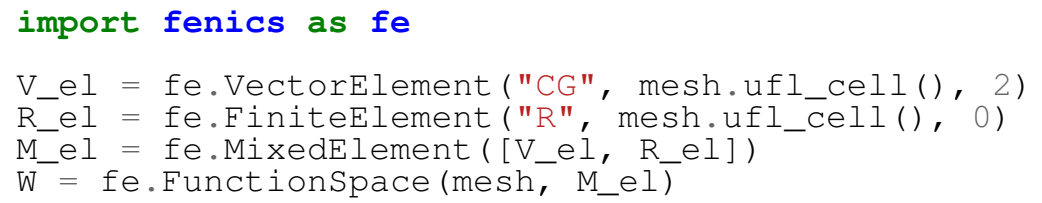

The first line create piecewise quadratic function space $\mathcal{V}$. The so called "Real" function space $\mathcal{R}$, created by the second line of code, represents one-value constant function over whole mesh. It brings additional global degree of freedom into governing matrices. The last two lines create mixed function space $\mathcal{W}=\mathcal{V} \times \mathcal{R}$. Now we can create tuple $\left\{\mathbf{u}, u_{0}\right\}$ of displacement trial function $\mathbf{u} \in \mathcal{V}$ and impactor trial function $u_{0} \in \mathcal{R}$ as one object of abstract space $\mathcal{W}$ and tuple of test functions $\left\{\delta \mathbf{u}, \delta u_{0}\right\} \in \mathcal{W}$ by following commands

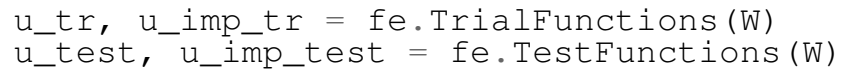

If we have trial and test functions, next step is forming of individual weak-form members. They reads

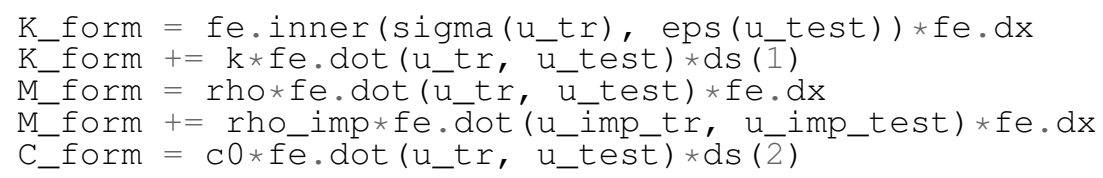

where rho_imp is impactor weight divided by domain volume.

The main advantage of FEniCS library is abstract approach to FEM modeling. The individual forms tied to individual matrices are written in python using UFL. Common operations, such as the dot or the inner product, are implemented simply by calling corresponding FEniCS functions fe.dot() or fe.inner(). Integration differential ds(1) represents $\Gamma_{\mathrm{k}}$ and $\mathrm{ds}(2)$ represents $\Gamma_{\mathrm{c}}$. Other implementation details are beyond the scope of the paper. Localization and assembling process is performed by function fe.assemble(). Therefore we can obtain individual matrices by

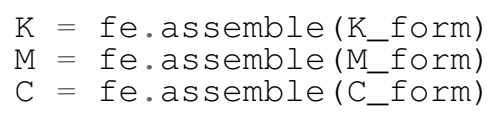

The last ingredient is the localization vector a for nonlinear Hertz law and localization matrix $\mathbf{A}=\mathbf{a a}^{\top}$ which enters to Jacobi matrix in Newton solver. The former one can be implemented as

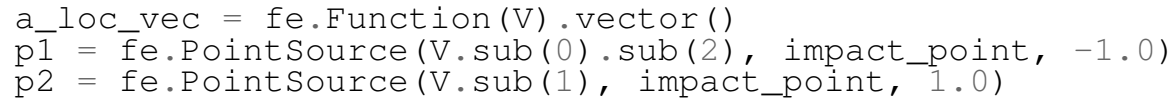


p1.apply (a_loc_vec)

p2.apply (a_loc_vec)

where impact_point is point of impact implemented through FEniCS object fe.Point () and object fe.PointSource() serves as the Dirac delta applicator. Physically it corresponds to application of concentrated force. The implementation of localization matrix $\mathbf{A}$ is more difficult, because the outer product $\mathbf{a a}^{\top}$ is not provided by FEniCS library (specifically by PETSc library) because it generally leads to dense matrix. The matrix $\mathbf{A}$ is therefore implemented by creating new PETSc matrix and using method setValues().

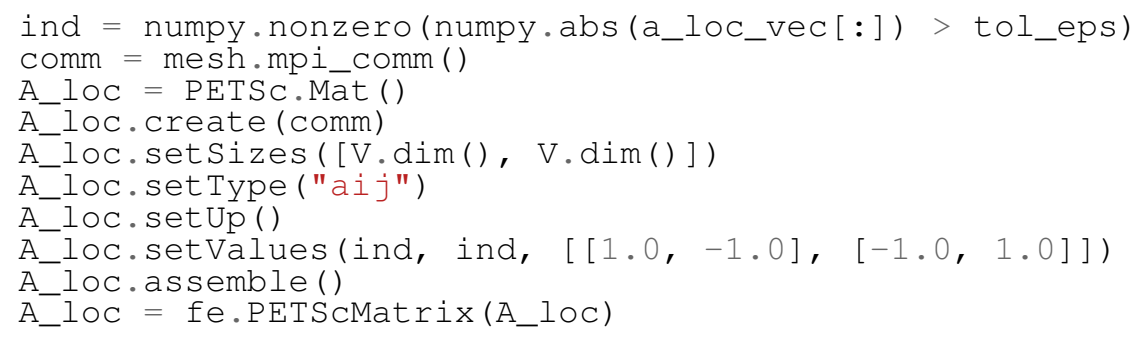

Array ind is the tuple of DoF indices, where nonlinear force is applied. It can be obtained by different way. We employ function nonzero from the numpy library. The rest of the code creates matrix $\mathbf{A} \in \mathbb{R}^{n \times n}$, where $n=\operatorname{dim}(\mathcal{W})$ and fills four selected components with values \pm 1 .

In this stage all matrices are assembled and the rest of the code depends on selected solver and on selected time integrator. This is no longer FEM problem, but a linear algebra problem. Therefore the concrete solver is not presented here.

\section{NUMERICAL RESULTS}

This section provides preliminary results for simulating glass plates impacted by external impactor. Glass is considered as linear isotropic material with Young's modulus $E_{1}=70$ GPa and Poisson's ratio $\nu_{1}=0.23$ according to standards [1], [7]. The stiffness of the contact follows from

$$
k_{0}=\frac{4}{3} \frac{R^{1 / 2}}{\frac{1-\nu_{1}^{2}}{E_{1}}+\frac{1-\nu_{2}^{2}}{E_{2}}},
$$

where material parameters $E_{2}=210 \mathrm{GPa}$ and $\nu_{2}=0.3$ of impactor was set as elastic steel material. Additionally, we set the radius of the impactor as $R=0.05 \mathrm{~m}$. Glass density is $\rho=2500 \mathrm{~kg} / \mathrm{m}^{3}$ according to [7] and impactor weight is $m_{\mathrm{imp}}=52 \mathrm{~kg}$.

We numerically test the solid glass plate where we prescribed boundary stiffness on the all faces except upper and lower surface. Response of individual plates are presented here through evolution of impactor acceleration. The so called soft impact, where weight of plate is higher order than impactor weight, is plotted in the left part of Fig. 1. The plate have dimensions $1.5 \times 1.5 \times 0.1 \mathrm{~m}$. There is evident minimum influence of support stiffness $k$. Different situation is in the right graph in Fig. 1, where evolution of impactor acceleration for plate with dimensions $1.5 \times 1.5 \times 0.04 \mathrm{~m}$ is displayed. It is evident that impactor hits the plate multiple times, but without some obvious pattern. If the boundary stiffness is negligible, the time of physical contact is small and the bodies bounce off each other with the possibility of small re-impact. With increasing boundary stiffness the behavior is more chaotic and impact time or number of impact is highly unpredictable. The same situation can be seen from Fig. 2. It represents further reduced plate with dimensions $0.5 \times 0.5 \times 0.02 \mathrm{~m}$ and total 

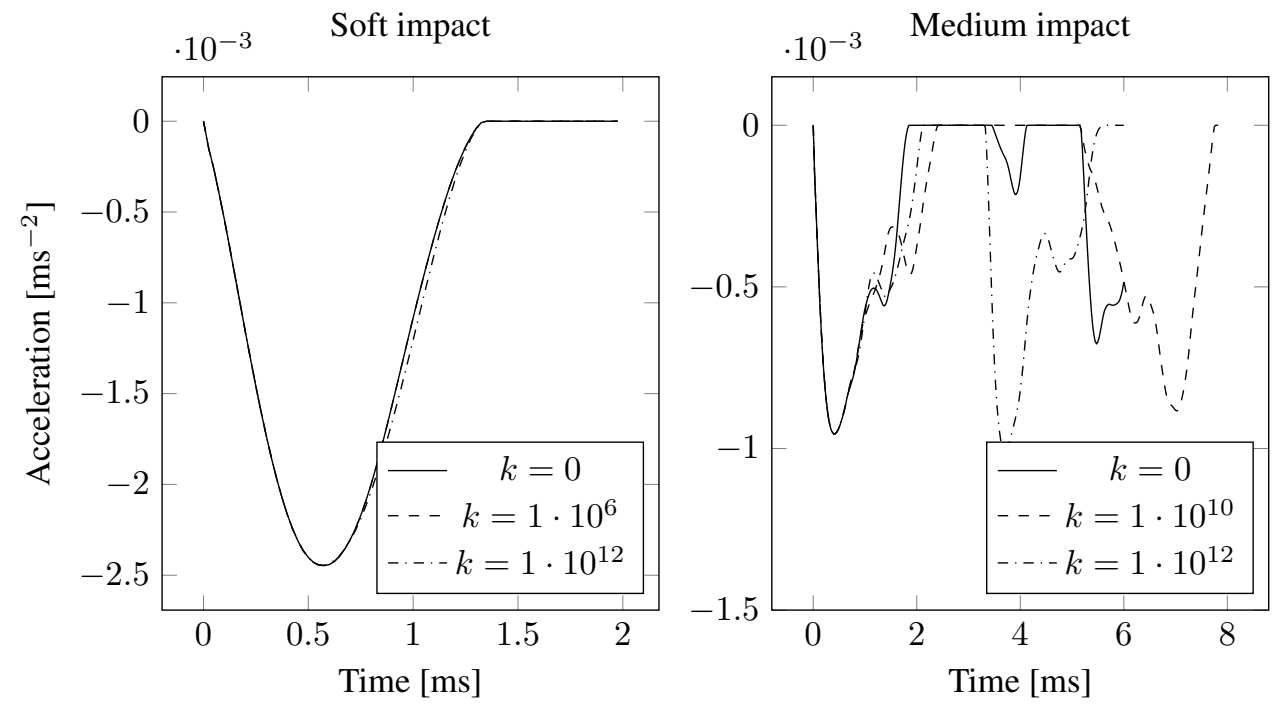

Figure 1: Evolution of impactor acceleration of glass plate $1.5 \times 1.5 \times 0.1 \mathrm{~m}$ (left) and $1.5 \times 1.5 \times 0.04 \mathrm{~m}$ (right) for different values of support stiffness $k$.

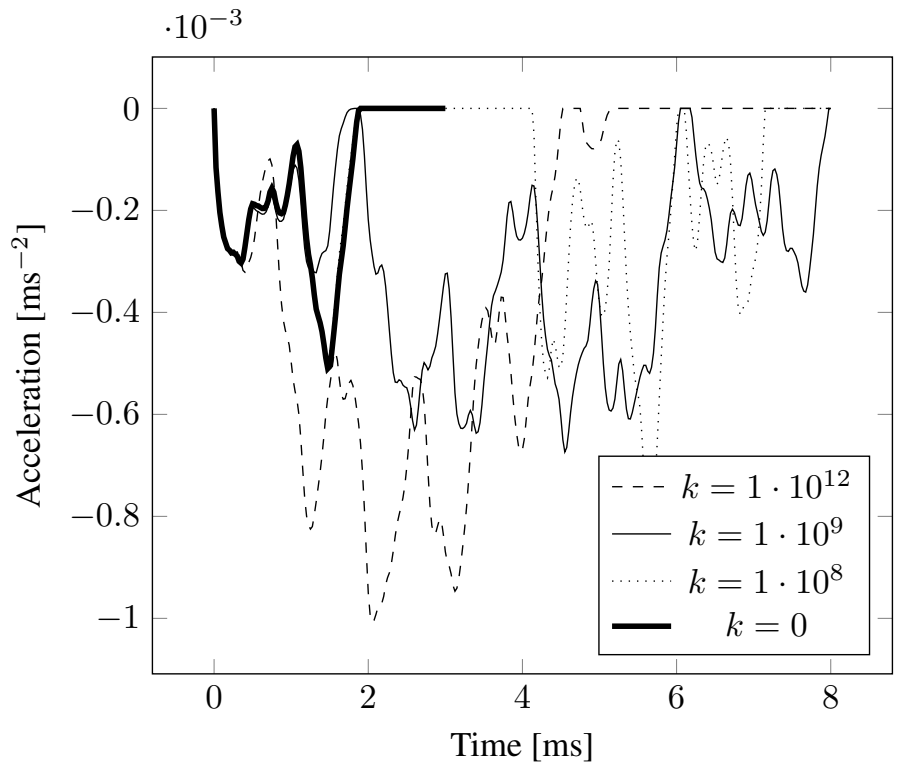

Figure 2: Evolution of impactor acceleration of glass plate $0.5 \times 0.5 \times 0.02 \mathrm{~m}$ for different values of support stiffness $k$. 
weight $12.5 \mathrm{~kg}$ (quarter of impactor weight). For example for zero stiffness there is short contact of plate and impactor, but if we introduce some stiffness of boundary, the first stage is the same, but second and even stronger impact appears later in diagram.

\section{CONCLUSION}

We introduced the implementation of impact analysis of two elastic bodies using the Hertz contact law. The emphasis was on implementation details in FEniCS finite element library, which offers abstract interface for solving PDEs. The numerical results show that even with simplified constitutive Hertz law, the impact is deterministically chaotic. This also applies for such a simple example as contact of glass plate with steel impactor. This is mainly due to the similar weights of both bodies and by large stiffnesses of body and impactor.

\section{ACKNOWLEDGEMENTS}

This publication was supported by the Czech Science Foundation, the grant No. 19-15326S and supporting by the Grant Agency of the Czech Technical University in Prague, grant No. SGS20/039/OHK1/1T/11, is also acknowledged.

\section{REFERENCES}

[1] Haldimann, M., Luible, A. \& Overend, M., Structural use of Glass, IABSE, 2008.

[2] Hertz. H., Uber die Beruhrung fester elastischer Korper. Journal fur die reine und angewandte Mathematik, 92, 156-171, 1881.

[3] Bourdin, B., Francfort, G. \& Marigo, J., The variational approach to fracture. Journal of Elasticity, 91(1-3), 2008.

[4] Alnæs, M. S. et al., The FEniCS Project Version 1.5. Archive of Numerical Software, 3(100), 2015.

[5] Spencer, A. J. M., Continuum Mechanics, Courier Corporation, 2012.

[6] Choi, I. H., Contact force history analysis of composite sandwich plates subjected to low-velocity impact. Composite Structures, 75(1-4), 582-586, 2006.

[7] CEN EN, 572-1: Glass in building-Basic soda lime silicate glass products-Part 1, 2004. 